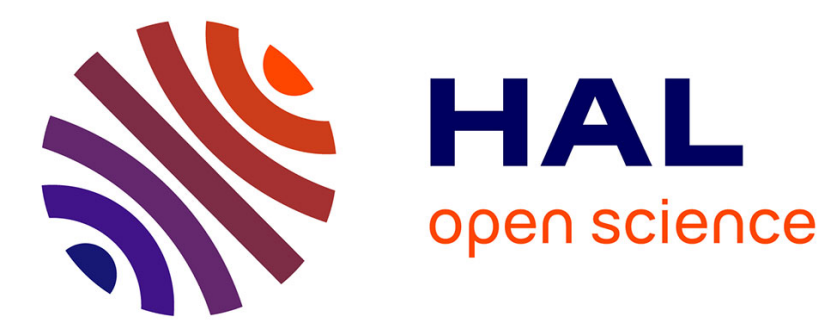

\title{
Free choice of unfunded systems: a first assessment
}

Gabrielle Demange

\section{To cite this version:}

Gabrielle Demange. Free choice of unfunded systems: a first assessment. 2005. halshs-00590861

\section{HAL Id: halshs-00590861 \\ https://shs.hal.science/halshs-00590861}

Preprint submitted on 5 May 2011

HAL is a multi-disciplinary open access archive for the deposit and dissemination of scientific research documents, whether they are published or not. The documents may come from teaching and research institutions in France or abroad, or from public or private research centers.
L'archive ouverte pluridisciplinaire HAL, est destinée au dépôt et à la diffusion de documents scientifiques de niveau recherche, publiés ou non, émanant des établissements d'enseignement et de recherche français ou étrangers, des laboratoires publics ou privés. 
Paris-Jourdan Sciences Economiques

48, BD JOURDAN - E.N.S. - 75014 PARIS

TEL. : 33(0) 143136300 - FAX : 33 (0) 143136310

www.pse.ens.fr

WORKING PAPER N² 2005 - 06

Free choice of unfunded systems: a first assessment

Gabrielle Demange

Codes JEL : H55, H87

Mots clés : unfunded systems, intragenerational redistribution, free choice 


\title{
Free choice of unfunded systems : a first assessment
}

\author{
Gabrielle DEMANGE ${ }^{1}$
}

July 22, 2005

\begin{abstract}
The first pillars of social security systems widely differ across European countries both in the contribution rate and intra-generational redistribution. What would be the impact of these differences if EU citizens had free access to all systems? This paper aims to highlight some basic features of this question in a very simple two countries model.

JEL : H55, H87
\end{abstract}

Keywords : unfunded systems, intra-generational redistribution, free choice

\footnotetext{
${ }^{1}$ EHESS, PSE 48 Boulevard Jourdan 75014, Paris, France. e-mail demange@pse.ens.fr. This is a revised version of WP 2005-06 PSE. The paper benefited from detailed comments of George Casamatta, Christian Gollier, Fabien Moizeau, and Pierre Pestieau. I also thank the seminar participants of the Cesifo conference "Strategies for reforming pension schemes" November 2004 for their remarks.
} 


\section{Introduction}

Most European countries have set up a mandatory unfunded pension scheme, often called first pillar, financed through contributions levied on wages. Although this common characteristic is crucial, the systems significantly differ in two dimensions at least. First, although benefit rules have evolved, systems can still be classified as they were at their set up : some are mostly "Bismarckian" with individuals' pensions that are earnings-related, while others are mostly "Beveridgean" with flat pensions. Second, the level of the mandatory contributions - hence the level of the pension benefits strongly varies across countries. For example, this level represented in 2003 roughly $9 \%$ of the GDP in the United Kingdom, 16,5\% in France, 19,5\% in Germany, and $32.7 \%$ in Italy ${ }^{2}$. Thus, the redistribution carried out within a generation and the level of the contributions are two major characteristics that differentiate European systems. Currently the minimal contributing period necessary to give pension rights is long, thereby limiting the "portability" of the systems. This limitation constitutes a barrier to workers' mobility, which may slow down labor integration, a major objective of the EU.

There are various ways to diminish the impact of such barriers. One is harmonization. Given the current differences in the systems and the problems of transition, agreement on a common system or even on steps toward convergence can only be slow. Another somewhat indirect but potentially

\footnotetext{
${ }^{2}$ Cross countries comparisons are however rather hazardous, and vary according to the definition of social security. In line with the objectives of the paper, I have tried to consider only the first pillars the systems. Data for France, Germany, and Italy are taken in http ://www.ssa.gov/policy/docs/progdesc/ssptw/2004-2005/europe/guide.html. The same document gives $23,8 \%$ for UK, but it includes the second pillar, which is also mandatory but funded. For a description of the UK system see the European Commission and the Council Joint report Adequate and sustainable Pensions (2003).
} 
powerful way to influence social security systems is "free choice". By free choice, I mean to let any EU citizen to choose the system of any EU country without moving. Owing to the differences in the social security taxes and the benefit rules, free choice could trigger a drastic change in the allocation of individuals between the various systems. Would all systems survive? What would be the impact on efficiency, redistribution, and ultimately on citizens welfare?

The purpose of this paper is to explore these questions in a model as simple as possible while still accounting for the basic features just outlined. The analysis is limited to two countries with identical fundamentals. Economies are modeled as overlapping generation models so as to discuss the trade-off between physical investment and direct inter-generational transfers such as performed by unfunded systems. To account for intra-generational redistribution, workers within a generation differ in their productivity. The growth rate of population and rate of return on investment are exogenous and constant over time.

An unfunded social security system is in place in each country, mandatory for its citizens. A system is characterized by two parameters, the contribution rate on earnings, and the "bismarckian" factor that determines the intra-generational redistribution operated by the system ${ }^{3}$. Even though the economies are identical, these parameters may differ in the two countries, to account of the stylized facts referred to above. I investigate the situation in which the citizens of both countries can freely choose either system, without having to move.

What effect may have free choice? Roughly speaking, the choice of an individual is determined by comparing the "rates of return" expected from each system (Aaron [1966]). Two factors influence this comparison. Not sur-

\footnotetext{
${ }^{3}$ I use here the modeling of Casamatta, Cremer and Pestieau (2000).
} 
prisingly, a first factor is related to the efficiency of inter-generational transfers (Samuelson [1958], Gale [1973]). If the growth rate of the population is less than the rate of return on investment for example, efficiency considerations favor the system with the lower contribution rate. The second factor is the redistribution operated within each system. In contrast to efficiency, redistribution affects individuals in a differential way according to their earnings. Furthermore the effective redistribution within a system is influenced not only by its design -bismarckian factor and contribution rate- but also by the distribution of earnings of its contributors (even a beveridgean system operates no redistribution if earnings are all equal). This is a crucial point to understand free choice since the contributors to each system are no longer determined by nationality.

Under free choice, individuals' choices affect the redistribution levels within each system, which in turn determine on individuals' choices. A simple example illustrates this interaction. Let contribution rates be equal in the two countries, one system be beveridgean and the other be bismarckian. Initially, in the absence of liquidity constraints, workers with wages smaller than the average one are better off in the beveridgean system than in the bismarckian. At the opening of the systems, presumably, low-income workers choose the beveridgean system, and the wealthy workers the bismarckian one (as is surely true if they base their choice on the initial situation). If this is the case however, the average contributors' earnings to the beveridgean system will diminish (and that to the bismarckian will raise). As a result, the effective redistribution within the beveridgean system decreases and the initial incentives to choose it is reduced.

To assess the full impact of free choice, this paper considers a steady state equilibrium. The wage distribution of the contributors to each system is constant overtime, determined by the choices of individuals who correctly 
expect the returns of each system for them (the so-called rational expectations hypothesis). I show that a (not necessarily unique) equilibrium always exists. Furthermore several types of equilibria may occur depending on whether one or both systems are active and which system is chosen by the high income workers. I investigate how the various parameters -characteristics of the systems, population growth rate, return to investment, wage dispersioninfluence the equilibrium type.

If both systems cannot be active in equilibrium, one system will be selected in the long run by all citizens and the other will be de facto eliminated. How to interpret this result? To suppose as in this paper that the opening of the systems would take place without any adjustment in their characteristics is not very realistic. The result nevertheless suggests that if the systems cannot be both active, adjustments must be sufficiently fast in order to avoid one system to be eliminated. Allowing for fast adjustments may be an even more unrealistic assumption owing to the current important differences between systems and the strong resistance to reforms.

The question addressed by this paper is political. The opening of systems limits the marges de manoeuvre of a "country". The analysis however differs from the approach initiated by Browning (1975), and referred to as the political approach to social security. Its purpose is to explain the characteristics of a system by considering various decision-making processes, such as planner, median voter, lobbies (see for example the review by Galasso and Porfeta (2002)). This paper clearly differs since its goal is to analyze the interaction between different systems taking the characteristics of the systems as "given", inherited from the past. On this respect, the closest analysis to ours is that of Casarico (2000). She also looks at the specific problem of integration and pension systems, with a focus that is somewhat complementary to ours. A more precise comparison is given after the analysis of the model. 
Also, the impact of national pay-as-you-go (payg) systems on the individuals' decisions to migrate has been examined by several authors (e.g. Hombourg and Richter [1993], Breyer and Kolmar [2002]). Individuals must contribute to the system where they live, and may differ only by a migration cost. Thus, in contrast with our analysis, redistribution is not an issue, and the driving force explaining why people move is the differences in (endogenous) population growth and interest rate across countries.

The plan of the paper is as follows. Section 2 introduces the model and Section 3 determines the initial situation when a mandatory system is in place within each country. Section 4 studies equilibrium configurations when the two systems are opened to the citizens of both countries. Some dynamics are considered in Section 5. Proofs are gathered in the final section.

\section{The model}

In what follows, I consider two countries, denoted by $A$ and $B$, with the same economy but with a different pension system.

\subsection{The economy in a country}

The economy in each country is described by the same overlapping generations model, with a structure close to that of Diamond (1965).

Each generation lives for two periods, there is a single good that can be either consumed or invested, and population grows at a constant rate $g-1$. An individual works only during the first period of his life, with an inelastic supply normalized to 1 .

The technology of production is linear with marginal productivities of capital and labor that are constant over time. The quantity of good available at date $t$ results from the return on investment at the previous period and 
from labor of the current young generation. An amount $s$ of capital invested in period $t-1$ will produce $r s$ units of good in period $t$ where $r$ is the exogenous return to investment. As for labor, workers differ in their productivity/wages $w$ : A $w$-worker denotes someone who produces and earns $w$. Wages are distributed on $\left[w_{\min }, w_{\max }\right]$ with a mean denoted by $\bar{w}$. Thus the total quantity of good available at date $t$ per head of old agents is $g \bar{w}+r s_{t-1}$ if $s_{t-1}$ was the average quantity invested in period $t-1$. The distribution of wages is assumed to be continuous, constant across generations.

Individuals' preferences bear on consumption levels when young and old, denoted by $c^{j}$ and $c^{v}$ (there is no altruism motive), and are strictly increasing in each argument. Preferences may be heterogeneous.

I shall assume away liquidity constraints. This assumption allows one to conduct the analysis without specifying preferences, by working with intertemporal wealth only. More precisely let us consider an individual who receives labor income net of contributions, $(1-\tau) w$, in the first period of his life, and expects to receive a pension benefit $\pi$ in the second period (dropping unnecessary time index). He faces the following successive constraints :

$$
c^{y}+s=(1-\tau) w \text { and } c^{o}=s r+\pi
$$

where $s$ is an investment if positive and a loan if negative. They imply :

$$
c^{y}+c^{o} / r=(1-\tau) w+\tau \frac{\pi}{r} .
$$

In other words, the discounted value of consumption levels is equal to the intertemporal wealth, defined as the value of net labor income plus the discounted rights to pension. Conversely, in the absence of constraint on $s$, the intertemporal constraint (2) describes all feasible consumption plans : (1) and (2) are equivalent. ${ }^{4}$

\footnotetext{
${ }^{4}$ The pension may not be correctly anticipated (even though we will require it to be at
} 
Thus, in the absence of liquidity constraints, the welfare of an individual varies as his intertemporal wealth. As a result, the impact of a pay-as-you-go system on an individual's welfare can be analyzed through the impact on wealth. Similarly, the choice between two systems is determined by comparing the wealth values expected from contributing to either system.

Remarks.

1. Growth in productivity/wages can be introduced in the usual way, by interpreting $g-1$ as the growth rate of the aggregate wage bill.

2. The assumption of a linear technology excludes endogenous variations in productivity, as would obtain with a non linear production function. Related to this, the absence of liquidity constraints makes sense only if aggregate savings are positive (see also footnote 6 ).

3. The ratio workers to retirees, equal to $g$, is exogenous. This ratio is however sensitive to some policies, especially to Social Security. Labor participation changes overtime, owing to changes in legislation affecting the choice of retirement date, or the number of working hours for instance. Also it has been suggested that life expectancy and fertility are influenced by Social Security (see Philipson and Becker [1983] and De la Croix and Doepke [2003] for instance). These aspects are not addressed here.

\subsection{Characteristics of a pension system}

At the "initial" situation, a pension system is in place in each country, mandatory for its citizens. Once systems are opened, each young individual will be able to choose between the two systems. This section describes the functioning of a system without specifying who contributes to it (and dropping unnecessary country index).

equilibrium). The only assumption that is needed is that the anticipation is single-valued : the young individual makes decisions as if he will receive $\pi$ for sure. 
A system is unfunded (payg), characterized by two parameters specifying the contribution rate, $\tau$, and the redistribution "bismarckian" factor $\alpha$.

Contributions are levied on wages, with a constant rate $\tau$ : a young $w$ worker contributes $\tau w$. By construction, the system is balanced. Thus, at date $t$, given $\bar{w}_{t}$ the average wage level of the contributors to the system and $g_{t}$ the number of contributors per pensioner, the average pension benefits per pensioner, $\bar{\pi}_{t}$, is equal to :

$$
\bar{\pi}_{t}=\tau g_{t} \bar{w}_{t} .
$$

The bismarckian factor determines the benefit rule, which relates the pension benefits of a specific pensioner to the contributions he made in the previous period. Let us consider a pensioner at $t$ who earned $w$ at period $t-1$ while the average wage over the contributors to the system was $\bar{w}_{t-1}$. He thus contributed $w / \bar{w}_{t-1}$ times the average level of contributions. If the bismarckian factor is $\alpha$ the pensioner receives benefits given by ${ }^{5}$

$$
\pi_{w, t}=\left(\alpha \frac{w}{\bar{w}_{t-1}}+(1-\alpha)\right) \bar{\pi}_{t}
$$

A pensioner whose contribution was equal to the average contribution per capita, $w=\bar{w}_{t-1}$, receives benefits equal to the average benefits per pensioner, $\bar{\pi}_{t}$, whatever value for $\alpha$. Note that for $\alpha=0$ all pensioners receive this level, independently of the amount of their previous contributions : the system is Beveridgean. At the opposite, a Bismarckian system obtains for $\alpha=1$, since pension benefits are proportional to contributions. Thus, for $\alpha$ between 0 and 1 , the system combines a Beveridgean system and a Bismarckian one. This is a crude description of the current systems, which are

\footnotetext{
${ }^{5}$ The benefit rule can also be written as
}

$$
\frac{\pi_{w, t}}{w}=\left(\alpha+(1-\alpha) \frac{\bar{w}_{t-1}}{w}\right) \frac{\bar{\pi}_{t}}{\bar{w}_{t-1}}
$$

which shows how replacement rates vary with income. 
much more complex (see for example the Whitehouse report on nine OECD countries).

\section{Pension systems}

Initially, each young worker contributes to the mandatory pension system of his country. I consider the steady state situation, in which the system is in place and is expected to remain in place. After examining a country, I draw some brief comparisons between distinct systems.

\subsection{National systems}

Let us consider a country with a system characterized by the parameters $(\tau, \alpha)$. While in place, the average level of wages of the contributors is $\bar{w}$. Also the numbers of contributors per pensioner is equal to $g$. This gives $g_{t}=g$, and $\bar{w}_{t-1}=\bar{w}_{t}=\bar{w}$ at a steady state, Therefore, from expressions (3) and (4), a $w$-worker will receive a pension benefit equal to $[\alpha w+(1-\alpha) \bar{w}] \tau g$. Plugging this value into (2) gives the value for intertemporal wealth :

$$
W(w)=\left[1+\tau\left(\frac{g}{r}-1\right)\right] w+\tau \frac{g}{r}(1-\alpha)(\bar{w}-w) .
$$

Note that in the absence of a payg system, wealth would be simply equal to the wage $w$. Thus, the system has a positive impact on an individual if $W(w)$ is larger than $w$. To highlight the impact of each characteristic, it is convenient to define

$$
R=1+\tau\left(\frac{g}{r}-1\right) \text { and } D=\tau \frac{g}{r}(1-\alpha) .
$$

With this notation, wealth writes as $W(w)=R w+D(\bar{w}-w)$.

The factor $R$ can be described as the rate of return of the system at the steady state situation : whatever value for $\alpha$, average wealth is equal to $R \bar{w}$ whereas, without a payg system, it would be equal to average wage $\bar{w}$. 
Furthermore, in the absence of redistribution, the wealth of a $w$-worker is given by $R w$ for any wage $w$. Thus, each individual benefits from the system ${ }^{6}$ if the rate $R$ is larger than 1 , that is if the growth rate of the population is larger than the rate of return on investment, and each one is hurt by the system in the opposite case of a rate $R$ less than 1 . In other words,

at steady states, a bismarckian payg system makes every indivi-

dual better off if $g>r$ and everyone worse off if $g<r$.

The distinction between the two cases is well known since Gale (1973), who referred to them respectively as the "Samuelson" and "classical" case.

In the presence of redistribution, the analysis remains valid "on average" since average wealth is $R \bar{w}$. In addition to $R w$, the wealth of a $w$-worker is affected by a term stemming from redistribution, $D(\bar{w}-w)$, positive for wages less than the average and negative otherwise. As a result, even if $g<r$, a system can nevertheless be beneficial to some low-income workers, or, at the opposite, even if $g>r$, a system can be detrimental to some high-income workers. Since the redistribution term is proportional to $D$, the factor $D$ determines the extent of the redistribution. Note that $D$ depends not only on the bismarckian factor, but also on the contribution rate and the ratio $g / r$.

\subsection{Comparing systems}

Even though the two countries, denoted by $A$ and $B$, have the same economy -identical population growth, return to investment, and wage distribution-

${ }^{6}$ The argument cannot be extended too much : it cannot be deduced from expression (6) that, if $g>r$, increasing the rate of contribution always leads to a Pareto improvement. Beyond a certain contribution rate, no young individual saves, which invalidates the approach by intertemporal wealth. To treat this question correctly, the return on capital must be endogenous, determined by a production function. Then, the rate of return becomes larger than population growth if saving/investment is sufficiently low. 
their systems may differ significantly, hence have a different impact on citizens welfare.

The characteristics of the system in country $I=A, B$ are denoted by $\left(\tau^{I}, \alpha^{I}\right)$. From (6), the rate of return to system $I$ and the extent of the redistribution are given by

$$
R^{I}=1+\tau^{I}\left(\frac{g}{r}-1\right) \text { and } D^{I}=\tau^{I} \frac{g}{r}\left(1-\alpha^{I}\right)
$$

and the wealth of a $w$-worker in country $I$ is

$$
W^{I}(w)=R^{I} w+D^{I}(\bar{w}-w)
$$

In the Samuelson case, $g>r$, the system that has the highest rate of return is the one with the largest contribution rate. In the classical case, it is the opposite. From now on, the system that has the highest rate of return will be referred to as the more efficient system. This is justified as follows.

In the absence of redistribution, the welfare of citizens in different countries but with the same wage $w$ is easily compared through their wealth $R^{I} w$. Hence, at the steady state with bismarckian systems, a $w$-worker is better off in the country that has the highest rate of return $R^{I}$.

In the presence of redistribution, the average wealth of the citizens is larger in the country with the largest return. Thus, with adequate transfers, all contributors to the less efficient system could be made better off by changing their contribution rate to that of the other country (that is decreasing it in the classical case, and increasing it in the Samuelson case). Note that there is an important difference between the two cases if one considers, instead of steady states, the transition from the less to the more efficient system. Whereas in the Samuelson case, every individual can be made better off, in the classical case, $g<r$, surely some individuals have to be hurt in a transition toward the more efficient system (by similar arguments as used in the seminal paper of Gale [1973]), 


\section{Equilibrium under free choice}

This section considers the situation in which each country opens its social security system to any citizen of the other country. More precisely, each young worker must contribute to a social security system, but can freely choose between the two systems without moving. The choice, necessarily made when young, is once for all.

To choose between system $A$ or $B$, a $w$-worker evaluates the wealth that he expects from each. Let us spell out this evaluation. The pension that is anticipated from a system depends on the wage level of its current and next contributors, and on the growth rate of the number of contributors, Current wages determine the future redistributive gains or losses within a system, and next wages together with the growth rate determine the level of pension benefits. Let $w_{t-1}^{I}$ and $w_{t}^{I}$ be the anticipated average wage of the young contributors to system $I$ at the current period $t-1$ and the subsequent period $t$, and $g_{t}^{I}-1$ be the anticipated growth rate of the number of contributors. According to (3) and (4), a $w$-worker will expect pension benefits equal to

$$
\left[\alpha^{I} \frac{w}{w_{t-1}^{I}}+\left(1-\alpha^{I}\right)\right] \tau^{I} g_{t}^{I} w_{t}^{I}
$$

from contributing to system $I$. This yields the level of intertemporal wealth :

$$
\left(1-\tau^{I}\right) w+\tau^{I} \frac{g_{t}^{I}}{r}\left[\alpha^{I} w+\left(1-\alpha^{I}\right) w_{t-1}^{I}\right] \frac{w_{t}^{I}}{w_{t-1}^{I}} .
$$

We look for a stationary equilibrium, which requires two conditions

(1) in each system, the number of contributors grows at a constant rate equal to that of the population, and the average wage of the contributors is constant over time, and

(2) individuals base their choices on these variables, which are correctly expected. 
Before making this definition more precise, it is convenient to analyze the choices of individuals who have (not necessarily correct) stationary expectations.

\subsection{The system that is more favorable to high-income}

The analysis of individuals' choices assuming stationary and identical expectations leads to a simple typology of the systems. Under stationary expectations, individuals expect the same types of workers to choose the systems at the current and next period. Thus, they expect the number of contributors to each system to grow as the population, $g_{t}^{I}=g$, and the average wage of the contributors to each system to be constant overtime $w_{t}^{I}=w_{t-1}^{I}$ for $I=A, B$. Let us denote by $w^{I}$ this constant expectation. From (9) and using the expressions (7) of $R^{I}$ and $D^{I}$, the intertemporal wealth expected by a $w$-worker from contributing to system $I$ is :

$$
W^{I}\left(w^{I}, w\right)=R^{I} w+D^{I}\left(w^{I}-w\right) .
$$

The choice of a system is made accordingly, by comparing the wealth values for $A$ and $B$, namely by the sign of

$W^{A}\left(w^{A}, w\right)-W^{B}\left(w^{B}, w\right)=\left[\left(R^{A}-D^{A}\right)-\left(R^{B}-D^{B}\right)\right] w+D^{A} w^{A}-D^{B} w^{B}$

The key point is that this expression is linear with respect to wage $w$, and that expected levels $w^{A}$ and $w^{B}$ do not affect the slope but only the level. Assume the slope to be positive (In the sequel, we exclude the degenerate case in which the slope is null). Then, if an individual prefers $A$ to $B$, all those who earn more than him also prefer $A$ to $B$. This leads to the following definition.

Definition. System $A$ is said to be more favorable to high-income than system $B$ if

$$
\left[R^{A}-D^{A}\right]-\left[R^{B}-D^{B}\right]>0
$$


which, replacing the $R^{I}$ and $D^{I}$ by their expressions, is equivalent to

$$
\tau^{A}\left(1-\frac{g}{r} \alpha^{A}\right)<\tau^{B}\left(1-\frac{g}{r} \alpha^{B}\right) .
$$

System $A$ is less favorable to high-income than $B$ if the inequalities are reversed. Under stationary and identical expectations, the workers who choose the system the more favorable to high-income are those whose wage is larger than a given threshold.

Which system is the more favorable is determined by the difference in efficiency, as measured by $R^{A}-R^{B}$, relative to the difference in the extent of redistribution, as measured by $D^{A}-D^{B}$. If both systems are bismarckian, there is no redistribution whatsoever, and the system the more favorable to high-income is the more efficient one. More interesting is the case of systems that differ in their redistribution. It is worth recalling that, in Europe, systems with rather flat benefits tend to be associated with low contribution rates. Thus consider the case where the system with the smaller bismarckian factor, say $B$, has the smaller contribution rate : $\alpha^{A}>\alpha^{B}$ and $\tau^{A}>\tau^{B}$. From (11), the system the more favorable to high-income is the one with the lowest product $\tau^{I}\left(1-\alpha^{I} g / r\right)$. Thus, under neutrality for example, $g=r$, the more bismarckian system $A$ is not necessarily the more favorable to highincome. As the ratio $g / r$ decreases, the more inefficient a payg system is, and the more likely it is that the system with the lower contribution rate is the more favorable to high-income workers.

\subsection{Example}

To illustrate this point, let us consider the case of France $(A)$ and United Kingdom $(B)$. The tax rate in UK is roughly half that in France, $\tau^{A} / \tau^{B} \approx 2$. Also, the UK system is much more redistributive than the french system. ${ }^{7}$

\footnotetext{
${ }^{7}$ This remark does not account for the reform which has just been decided in France. Indeed the minimum level for pension benefits has been increased up to $85 \%$ of the mini-
} 
According to some data, the parameters $\alpha^{A}=0.8$ and $\alpha^{B}=0.2$ are reasonable. The threshold value of $g / r$ that determines whether $A$ is more favorable to high income than $B$ is $1 / 1.4 \approx 0.7$. This gives that

for $g / r>1, A$ is more efficient and more favorable to high income than $B$, for $1>g / r>0.7, A$ is less efficient but more favorable to high income than $B$, and for

$g / r<0.7, A$ is less efficient and less favorable to high income than $B$.

Thus, the UK system, although much more redistributive than the french system, can be more favorable to high-income thanks to its low contribution rate. This is especially true if payg systems are perceived as inefficient.

Not surprisingly, the ratio of growth rate to investment return plays a crucial role. Which value for this ratio is reasonable? This is quite a delicate question because it is not clear which return should be chosen for $r$. A period here represents roughly thirty years. If one takes for $r$ the return on the stock market since the second world war, and for $g$ the projected growth rate of aggregate wage bill, the compounding effect will give a low value for $g / r$. This is however related to the equity premium puzzle. If indeed individuals are risk averse and ready to pay a high risk premium, then one should take for $r$ a much smaller value than the stock market return. Also, a payg system provides retirees with an annuity, thereby insuring them against the risk of living old. Making insurance compulsory avoids the usual problems encountered in markets with asymmetric information. As documented by various studies, the premium associated to the longevity risk is roughly $5 \%$ (see Brown, Mitchell, and Poterba [2001]). To account for this premium, an extra return on a payg could be introduced. Due to these difficulties and the uncertainty on future, I shall discuss in next section equilibria for different mal wage. For a rather large fraction of low-income earners, this constraint may become binding, which would make the French system more beveridgean than previously. I thank Thomas Piketty for having mentioned this point to me. 
values of $g / r$.

\subsection{Equilibrium}

Given an anticipated average wage $w^{I}$ of the contributors to each system $I$, let $w^{*}$ be the wage level defined by $W^{A}\left(w^{A}, w^{*}\right)-W^{B}\left(w^{B}, w^{*}\right)=0$. To fix the idea, assume system $A$ to be more favorable to high-income. The individuals who choose system $A$ are those who earn more than $w^{*}$. Note that the threshold $w^{*}$ may not be in the range of wages. If $w^{*} \leq w_{\min }$ for example, all individuals choose $A$, and if $w^{*} \geq w_{\max }$, all choose $B$.

Individuals' anticipation on contributors' average wages determine their choices, which in turn determine the realized wages. To get an equilibrium, anticipation and realization must be consistent. This leads to the definition :

Definition. Let system $A$ be more favorable to high-income than B. An equilibrium is determined by average wages $w^{A}$ and $w^{B}$, and $w^{*}$ that satisfy $W^{A}\left(w^{A}, w^{*}\right)-W^{B}\left(w^{B}, w^{*}\right)=0$ and the expectations conditions :

- if $w^{*}$ is in $] w_{\min }, w_{\max }\left[: w^{A}=E\left[w \mid w \geq w^{*}\right], w^{B}=E\left[w \mid w \leq w^{*}\right]\right.$

both systems are active and the equilibrium is called an AB-equilibrium

- if $w^{*} \leq w_{\text {min }}: w^{A}=\bar{w}, w^{B}=w_{\text {min }}$

only system $A$ is active and the equilibrium is called an A-equilibrium,

- if $w^{*} \geq w_{\max }: w^{A}=w_{\max }, w^{B}=\bar{w}$

only system $B$ is active and the equilibrium is called a $B$-equilibrium.

For an active system, the expectation condition says that the anticipation of the average wage of the contributors to an active system is "correct" equal to its expectation conditional on individuals' choices. ${ }^{8}$ For an inactive

\footnotetext{
${ }^{8}$ Note that the wage distribution being identical in the two countries, the wage distribution in the union of the two countries is identical to that of a single country.Thus, if only $I$ is active, $w^{I}=\bar{w}$.
} 
system, the condition needs a justification : since there are no contributors, the conditional expectation of their average wage is not well defined. Hence individuals' behaviors are supported by some "beliefs" about this wage. ${ }^{9}$ In the above definition, the beliefs are those justified by a perfect equilibrium argument. If the set of contributors to $B$ is small for example, it is formed by the individuals whose wages are close to the minimum. Taking the limit, if only $A$ is active, the belief on $w^{B}$ is the minimum wage. A similar argument justifies that with only $B$ active, the belief on $w^{A}$ is set to the maximum wage.

Before going further it is helpful to note that the system the less favorable to high-income is eliminated whenever it is also the less efficient. The intuition is clear. Suppose there is an $A B$-equilibrium and consider a $w^{*}$ worker who is indifferent between $A$ and $B$ (a similar argument shows that a $B$-equilibrium does not exist by considering workers with wage $w_{\max }$ ). By choosing $A$, the worker would benefit from the larger efficiency return provided by $A$. Furthermore, since the wage $w^{*}$ is not greater than $w^{A}$, he can only benefit from redistribution in $A$ instead of being penalized by it in $B$ : he definitely prefers $A$ to $B$, a contradiction. The analysis is more complex if, from the point of view of low-income workers, efficiency and redistribution benefits enter into conflict. The following proposition characterizes the equilibrium configurations in function of the parameters.

Proposition 1. Let system $A$ be more favorable to high-income than $B$. There exists

\footnotetext{
${ }^{9} \mathrm{~A}$ referee objects that a young individual can choose a system that has no retirees, say $B$, and get pension benefits when old for free. It is true only if next period, a young will indeed agree to contribute to $B$. Looking at intertemporal wealth, which amounts at considering stationary behavior, precisely accounts for this requirement.
} 
- an A-equilibrium if and only if

$$
W^{A}\left(\bar{w}, w_{\min }\right)-R^{B} w_{\min } \geq 0 \Leftrightarrow R^{B}-R^{A} \leq D^{A} \frac{\bar{w}-w_{\min }}{w_{\min }}
$$

- a B-equilibrium if and only if

$$
W^{B}\left(\bar{w}, w_{\max }\right)-R^{A} w_{\max } \geq 0 \Leftrightarrow D^{B} \frac{w_{\max }-\bar{w}}{w_{\max }} \leq R^{B}-R^{A}
$$

- an AB-equilibrium if

either an $A$ and a $B$ equilibrium both exist : (12) and (13) hold

or no one exists : neither (12) nor (13) holds.

It follows that an equilibrium always exists. As already said, it can only be an $A$-equilibrium if $A$ is more efficient : (12) holds but not (13) if $R^{B} \leq R^{A}$. Otherwise, the trade-off between efficiency and redistribution for low income or top income workers determines equilibrium configurations. To see this, let us explain how the equilibrium conditions are obtained. To check whether $A$ alone can be in equilibrium, assume that $A$ is chosen by every worker. The average wage $w^{A}$ is equal to the overall mean $\bar{w}$. To form an equilibrium, it suffices that workers whose wages are close to the minimum level $w_{\min }$ have no incentives to subscribe to $B$. This gives condition (12), which results from the following trade-off. By subscribing to $B$, on one hand $w_{m i n}$-workers lose all the redistribution benefits in $A$ without getting any in $B$ (because wages in $B$ are roughly identical), but on the other hand they benefit from the larger return in $B$ (assumed to be more efficient). If the loss outweighs the efficiency gain, an $A$-equilibrium is obtained. Since the larger the ratio $\bar{w} / w_{\min }$ is, the larger the loss in redistributive benefits, a low value for the minimum wage makes more likely an $A$-equilibrium to exist. Similarly a large value for the extent of the redistribution in $A$ (subject of course to $A$ be more favorable to high income) makes more likely an $A$-equilibrium to exist. 
Similarly, a $B$-equilibrium is obtained if workers whose wages are close to the maximum level $w_{\max }$ have no incentives to subscribe to $A$, which gives condition (13). The condition is strong : it requires that top income workers are better off by subscribing to system $B$ applied to the whole population rather than by subscribing to $A$ without redistribution loss. As the ratio $w_{\max } / \bar{w}$ and the extent of the rredistribution in $B$ increase, the redistribution losses for a top income worker subscribing to $B$ rather than to $A$ outweighs the efficiency gains : $B$ alone is in equilibrium only for a small enough ratio and small value for $D^{B}$.

Note that the above arguments are valid whatever the assumption on the distribution of earnings, whether continuous or not : only the incentives of the top or bottom income workers matter. ${ }^{10}$ This insight is likely to be quite robust and to extend to more general benefit rules.

According to this discussion, as the range of wages is enlarged, the redistribution effects become predominant and determine the equilibrium. Workers who most benefit from redistribution and those who are the more penalized by it are both encouraged to choose the system the more favorable to high income : for sufficiently low $w_{\min }$ and sufficiently large $w_{\max }$, only condition (12) holds. This gives :

Corollary. The system the more favorable to high-income is the only active at equilibrium if the range of wages is sufficiently large.

Instead, various equilibrium configurations are possible when the dispersion of wages is not too large and the system the less favorable to high income is the more efficient. Even the three types of equilibrium can simultaneously

\footnotetext{
${ }^{10}$ With a discrete distribution, the condition for an $I$-equilibrium to exist is identical. The $A B$ equilibria may be a little bit different : some may be semi-pooling, meaning that individuals with the same wage choose distinct systems (of course they must be indifferent between both.
} 
exist. As long as redistribution or efficiency is not dominant factor, the incentives conditions, as given by (12) and (13) are to some extent independent : one bears on incomes at the bottom and the other at the top. This is illustrated by the subsequent example.

\subsection{Example (continued)}

Consider again the illustrative case in which $\alpha^{A}>\alpha^{B}$ and $\tau^{A}>\tau^{B}$. We know that when one system is both more efficient and more favorable to high income than the other, it is the only active at equilibrium. It immediately follows that only the more beveridgean system $B$ is active if payg systems are sufficiently inefficient, while it is the more bismarckian system $A$ if they are sufficiently efficient. ${ }^{11}$ Taking the values of the France-UK example in Section 2, this gives that

- for $g / r>1$, an $A$-equilibrium obtains because system $A$ is more efficient and more favorable to high income than $B$,

- for $g / r<0.7$, a $B$-equilibrium obtains because $A$ is less efficient and less favorable to high income than $B$.

It remains to determine what happens when efficiency and redistribution enter into conflict, which occurs here for $1>g / r>0.7: A$ is more favorable to high-income but less efficient than $B$. The case $g / r=0.8$ is illustrated in figure 1.

In the top graph, the dashed line represents $R^{B} w-W^{A}(\bar{w}, w)$ as a function of $w$. According to (12), there is an $A$-equilibrium if it is negative at $w_{\text {min }}$, that is if $w_{\text {min }}<a \approx 0.6$. Similarly, the normal line represents $W^{B}(\bar{w}, w)-R^{A} w$, and according to (13), there is a $B$-equilibrium if it is positive at $w_{\max }$, which gives $w_{\max }<b \approx 1.5$. The difference in wealth, at

\footnotetext{
${ }^{11}$ The first case holds if both inequalities, $g<r$ and $\tau^{B}\left(1-\alpha^{B} g / r\right)<\tau^{A}\left(1-\alpha^{A} g / r\right)$ hold, and the second if both inequalities are reversed.
} 

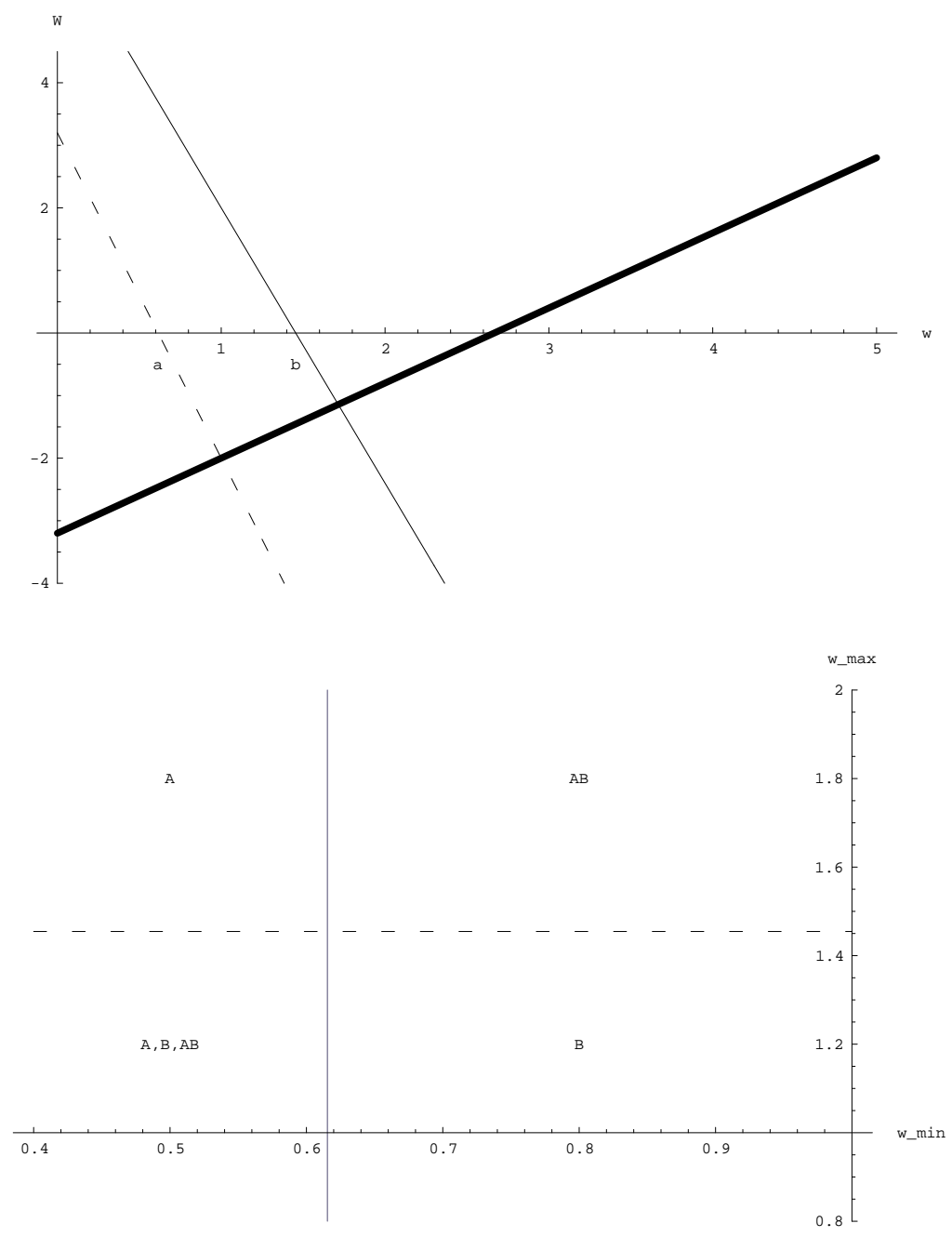

FIG. 1 - Parameters : $\tau_{A} / \tau_{B}=2, \alpha^{A}=0.8, \alpha^{B}=0.2, g / r=0.8, \bar{w}=1$.

Top graph: The horizontal axis represents $w$, the vertical one differences in wealth, multiplied by 100 . The thick line represents $W^{A}(\bar{w},)-.W^{B}(\bar{w},$.$) ,$ the dashed line $R^{B} w-W^{A}(\bar{w}, w)$ (there is an $A$-equilibrium if $w_{\min }<a \approx$ 0.6 ) and the normal line : $W^{B}(\bar{w}, w)-R^{A} w$ (there is a $B$-equilibrium if $\left.w_{\max }<b \approx 1.5\right)$

Bottom graph : Equilibria as function of $w_{\min }$ and $w_{\max }$ 
the initial situation, $W^{A}(\bar{w}, w)-W^{B}(\bar{w}, w)$ is also represented (the thick line). This is useful for subsequent welfare comparisons and for understanding the initial incentives to choose one system rather than another. Here the difference is increasing in $w$ because $A$ is more favorable to high income than $B$. Also it is negative at the mean value $\bar{w}$ because $B$ is more efficient than $A$ and $W^{I}(\bar{w}, \bar{w})=R^{I} \bar{w}$.

The bottom graph summarizes the equilibrium types as a function of the range of earnings keeping the mean constant. When the range is small $\left(w_{\min }>0.6\right.$ and $\left.w_{\max }<1.5\right)$, there is only a $B$-equilibrium : efficiency effects are dominant. One checks that for these values, at the initial situation, all citizens in $A$ would prefer system $B$ : the difference $W^{A}(\bar{w}, w)-W^{B}(\bar{w}, w)$ is negative in the relevant range for $w$. This is always true as we shall see in Proposition 2. As the range is increased, we move to the north west and only the $A$-equilibrium remains : the redistribution effects become dominant.

\subsection{Welfare}

In light of these results, one may wonder whether introducing free choice is beneficial. To avoid considering too many cases, I discuss the situation in which an $I$-equilibrium is obtained (i.e. I do not consider an $A B$-equilibrium). Then the winners or losers are easily determined. To see this, note that any $w$-worker gets an intertemporal wealth equal to $W^{I}(\bar{w}, w)$. Thus, citizens in country $I$ are not affected by the reform while in $J$ the losers (resp.winners) are those for whom the intertemporal wealth $W^{J}(\bar{w}, w)$ was larger (resp. smaller) than $W^{I}(\bar{w}, w)$.

To fix the idea let $A$ be more favorable to high income. Then, using that $W^{I}(\bar{w}, \bar{w})=R^{I} \bar{w}$, the following inequalities hold

$$
\begin{array}{rll}
\left(R^{A}-R^{B}\right) \bar{w} & >W^{A}(\bar{w}, w)-W^{B}(\bar{w}, w) & \text { for } w<\bar{w} \\
\text { and }\left(R^{A}-R^{B}\right) \bar{w} & <W^{A}(\bar{w}, w)-W^{B}(\bar{w}, w) & \text { for } w>\bar{w}
\end{array}
$$


If $A$ is not less efficient than $B$, there is a $A$-equilibrium only. Also, surely in country $B$ all citizens whose income is larger than $\bar{w}$, are made better off from (15) since $R^{A}-R^{B} \geq 0$. Of course, other citizens are better off, maybe all if efficiency gains are large enough relative to redistribution effects.

Assume now that $A$ is less efficient than $B$.

If a $B$-equilibrium obtains, the opening of the systems is beneficial without ambiguity, as stated by the following proposition.

Proposition 2. The system the more favorable to high-income can be eliminated only if, at the initial situation, all individuals are better off with the other system : If $A$ is more favorable to high-income a B-equilibrium exists only if

$$
W^{B}(\bar{w}, w) \geq W^{A}(\bar{w}, w) \text { for any } w \text { in }\left[w_{\min }, w_{\max }\right]
$$

and thus leads to a (weak) Pareto improvement over the initial situation.

Condition (16) is strong but it is not a sufficient condition for a Bequilibrium to exist. The range of wages must be small enough. Consider the example again. If $1.5<w_{\max }<2.8$ and $w_{\min }<0.6$, only the $A$-equilibrium exists, but $B$ applied to the whole population gives a larger wealth to every one than $A$.

Instead, if the $A$-equilibrium obtains, some $B$-citizens must lose since the less efficient system is now in place in country $B$. In particular, from (14) and the fact that $R^{A}<R^{B}$, all workers who earn less than the average wage are worse off. Since the average wage is typically larger than the median value, more than a majority of workers in $B$ are made worse off by the reform. How can this happen? The dynamics contemplated below helps to understand this point : Assuming that initially all workers who prefer $B$ choose it, system $A$ becomes more attractive, which triggers new choices, which may eventually lead to all choosing $A$. Actually it can even happen that 
all workers in $B$ are hurt at a $A$-equilibrium. In that case, implementing $B$ for everybody would be weakly Pareto improving. This occurs if (16) is met but the $A$-equilibrium obtains. This is surely the case if the $A$-equilibrium is unique $\left(1.5<w_{\max }<2.8\right.$ and $w_{\min }<0.6$, in the example).

To sum up, even though the model is very simple, the welfare impact of the opening of the systems largely depends on the situation at hand. If redistribution losses or gains are too important, there is few chances that a Pareto improvement will be obtained.

At this point, it is worth comparing our analysis with Casarico (2000). In two countries with identical economies, compulsory pension systems are in place, unfunded in one country and fully funded in the other. Redistribution plays no role (the analysis is conducted with a representative individual in each country). The paper focuses on the impact of these differences on capital integration, when capital becomes fully mobile, labor remaining immobile. Production is carried out through a neoclassical production function. Before capital integration, owing to the different pension systems, investments, hence their returns, differ in the two countries. Capital integration has a welfare effect because the return to investment is equalized across countries. While this effect is clearly absent in our analysis, Casarico does not allow workers to choose a system. If they could, they would all choose the more efficient (since there is no redistribution).

\section{Dynamics}

By keeping the tax rate constant, the balance of a system is ensured through adjustments in pension benefits. As a result, a system can be thought of as a defined contribution one. In a correct expectations framework, as just considered, it is also a defined benefit one. It may no longer be 
true under some dynamics. Dynamics are determined by expectations and information. At the time workers have to choose a system, they are concerned with the wage level of the current and next contributors to each system : The current ones determine the future redistributive gains or losses within a system, and the next ones give the level of pension benefits. Starting from the initial situation in which each system in place in a country is exclusively for the citizens, I consider here the evolution of the systems driven by myopic expectations.

Myopic expectations mean that workers at time $t$, who do not know yet the choice of their contemporaries and descendants, expect that they will perform the same choice as the previous generation. For example, initially, all workers assume the average contributors wage to be identical in each system, equal to $\bar{w}$. A threshold value $w_{0}^{*}$ for wages is determined, according to which all workers with income larger (resp. smaller) than the threshold choose $A$ (resp. $B$ ), still assuming $A$ more favorable to high-income than $B$. Afterwards, the evolution of the system is described as follows. Let $w_{t-1}^{*}$ be the threshold value between $A$ and $B$ at time $t-1$. Workers at time $t$ observe this value. Under myopic expectations, they expect the choice of current and next contributors to remain unchanged. Thus, they expect the average wage level of the current and next contributors to each system to be given by :

$$
w_{t-1}^{A}=E\left[w \mid w \geq w_{t-1}^{*}\right], w_{t-1}^{B}=E\left[w \mid w \leq w_{t-1}^{*}\right] .
$$

It follows that a $w$-worker at date $t$ evaluates the wealth generated by system $I$ as $W^{I}\left(w_{t-1}^{I}, w\right)$ defined by (10), and chooses between $A$ and $B$ accordingly. A new threshold $w_{t}^{*}$ level is determined, and so on.

Proposition 3 Let system $A$ be more favorable to high-income than $B$. Assume myopic expectations. Dynamics converge to 
the (unique) $A$ equilibrium if $A$ is more efficient than $B$, in one step to the $B$ - equilibrium if it exists.

Otherwise, assuming that $D^{B} E[w \mid w \leq x]-D^{A} E[w \mid w \geq x]$ is non-decreasing, dynamics converge to an equilibrium.

In the first two cases convergence always occurs. Note that the $B$-equilibrium Pareto dominates the $A$-equilibrium if both exist (by Proposition 2). Therefore a "good" equilibrium is selected. In other cases, an additional assumption is needed. To understand why, consider the opening of the systems in which workers choose the systems assuming the same distribution within each one. Since those who choose $B$ are less wealthy than those who choose $A$, the redistribution within the system $B$ is diminished and within $A$ increased : more individuals will choose $A$ next period. The monotonicity assumption ensures that the set of workers who choose $A$ will grow. The assumption is satisfied if $D^{A}$ is sufficiently low, $A$ bismarckian for instance, or if wages are uniformly distributed. ${ }^{12}$

\section{Concluding remarks}

Even though the model is too simple in many dimensions, it helps to highlight some features that are likely to be quite robust. First, the analysis shows that the system that is preferred by high income workers is not necessarily the more bismarckian one. Both the levels of the contribution rates and the efficiency or inefficiency of unfunded systems play an important role. In particular, in situations in which unfunded are perceived as very inefficient, the system with the lower contribution rate is preferred. Second, a

\footnotetext{
${ }^{12}$ Since $A$ is less efficient and more favorable to high income than $B$, both inequalities $0>$ $R^{A}-R^{B}>D^{A}-D^{B}$ hold. Thus the monotonicity condition is met if the slope of $E[w \mid w \geq$ $x]$ is not larger than that of $E[w \mid w \leq x]$. This is true for a uniform distribution since the conditional expectations aare respectively equal to $\left(w_{\max }+x\right) / 2$ and $\left(x+w_{\min }\right) / 2$.
} 
large dispersion of wage earnings eliminates the system the less favorable to high income even it is the more efficient : the redistribution effects become dominant for the workers who most benefit from redistribution or those who are the more penalized by it. Third, free choice does not necessarily lead to select the more efficient system. In some cases, the new situation may be pareto dominated by the initial one.

The analysis has been conducted under strong simplifying assumptions. It should be extended in several directions in order to test the robustness of the results. First production and endogenous factor prices could be introduced. Second, uncertainty on production and population growth would make the comparison between rates of return of the systems less trivial and more realistic. Liquidity constraints, which are likely to be binding on low income workers, should be taken into account.

Finally, to incorporate political elements in the analysis would be of course most interesting. It would require to describe the adjustments of the systems confronted with the impact of free choice, even if such adjustments can only be slow. The analysis would then be similar in some aspects to that of "fiscal competition" (see for example Epple and Romer (1991), Wildasin (1991)). A basic concern is whether factor mobility, as dictated by the european construction, necessarily undermines redistributive policies. Not surprisingly, the literature on taxation between areas -regions, jurisdictions, countries- and the limitation to redistribution due to the mobility of capital or labor, is vast and is still growing (see the survey of Cremer and Pestieau (2002). 


\section{Proofs}

Recall that under the assumption of stationarity, the wealth of a $w$ worker contributing to system $I$ with expectations $w^{I}$ is given by (10) :

$$
W^{I}\left(w^{I}, w\right)=R^{I} w+D^{I}\left(w^{I}-w\right) .
$$

Proof of Proposition 1 Let us determine the conditions under which there is an active equilibrium with only $A$. In this case $w^{A}=\bar{w}$ and $w^{B}=w_{\min }$. An equilibrium is obtained if and only if an individual whose wages are minimum is not incited to choose system $B$. This is written as $W^{A}\left(\bar{w}, w_{\text {min }}\right)-W^{B}\left(w_{\text {min }}, w_{\text {min }}\right) \geq 0$. Using the expression of wealth (10), this gives the inequality (12).

In a similar way, a situation with only $B$ active forms an equilibrium if an individual whose wage is maximal is not incited to choose system $A$. Since $w^{A}=w_{\max }$ and $w^{B}=\bar{w}$, this gives $W^{A}\left(w_{\max }, w_{\max }\right)-W^{B}\left(\bar{w}, w_{\max }\right) \leq 0$ which yields (13).

It remains to consider an $A B$-equilibrium. Let the function $\phi$ be defined on $\left[w_{\min }, w_{\max }\right]$ by

$$
\phi(x)=W^{A}(E[w \mid w \geq x], x)-W^{B}(E[w \mid w \leq x], x) .
$$

By continuity of the distribution of wages, the function $\phi$ is continuous. An equilibrium with two active systems is associated with $w^{*}$ in $] w_{\min }, w_{\max }[$ that satisfy $\phi\left(w^{*}\right)=0$. Note that (12) is equivalent to $\phi\left(w_{\text {min }}\right) \geq 0$ and (13) to $\phi\left(w_{\max }\right) \leq 0$. By continuity of $\phi$ it follows that if no $I$ equilibrium exists, there is an interior $w^{*}$ such that $\phi\left(w^{*}\right)=0$. Similarly, if both equilibria exist and the inequalities are strict, there is also an $A B$-equilibrium

Proof of Proposition 2. As just seen, a $B$-equilibrium exits iff $W^{B}\left(\bar{w}, w_{\max }\right)-$ $W^{A}\left(w_{\max }, w_{\max }\right) \geq 0$. Since $W^{A}\left(w_{\max }, w_{\max }\right)=R^{A} w_{\max } \geq W^{A}\left(\bar{w}, w_{\max }\right)$ (because of the redistribution loss), this gives $W^{B}\left(\bar{w}, w_{\max }\right)-W^{A}\left(\bar{w}, w_{\max }\right) \geq$ 
0 which implies $W^{B}(\bar{w}, w)-W^{A}(\bar{w}, w) \geq 0$ for any $w$ (because $B$ is less favorable to high income).

Proof of Proposition 3. To simplify notation, set $\Delta=R^{A}-R^{B}-D^{A}+D^{B}$. The assumption that system $A$ is more favorable to high-income than $B$ writes as $\Delta>0$. The choice of a $w$-worker at $t$ is made on the basis of the difference in expected wealth

$$
W^{A}\left(w_{t-1}^{A}, w\right)-W^{B}\left(w_{t-1}^{B}, w\right)=\Delta w+D^{A} w_{t-1}^{A}-D^{B} w_{t-1}^{B}
$$

where the values $w_{t-1}^{A}$ are $w_{t-1}^{B}$ are the average wages of the contributors to each system observed at time $t-1$. By positivity of $\Delta$, the function is increasing in $w$. Thus, all workers whose wage is larger than $\left(D^{B} w_{t-1}^{B}-D^{A} w_{t-1}^{A}\right) / \Delta$ choose $A$ and the others choose $B$. If this wage value is larger than $w_{\max }$, then everybody chooses $A$, and if it is smaller than $w_{m i n}$, everybody chooses $B$. In order to define the threshold $w_{t}^{*}$, it is convenient to consider the projection $P$ on the interval $\left[w_{\min }, w_{\max }\right]$ :

$$
\begin{aligned}
P(x) & =w_{\min } \text { if } x<w_{\min }, \\
& =x, x \in\left[w_{\min }, w_{\max }\right], \\
& =w_{\max } \text { if } x>w_{\max }
\end{aligned}
$$

The threshold $w_{t}^{*}$ is defined by

$$
w_{t}^{*}=P\left(\left(D^{B} w_{t-1}^{B}-D^{A} w_{t-1}^{A}\right) / \Delta\right) .
$$

The values $w_{t-1}^{A}$ are $w_{t-1}^{B}$ expected at $t$ are based on observed at time $t-1$. Thus, at the opening of the systems, $t=0$, both are equal to the average wage :

$$
w_{-1}^{B}=w_{-1}^{A}=\bar{w}
$$

and afterwards, $t>0$, they satisfy :

$$
w_{t-1}^{A}=E\left[w \mid w \geq w_{t-1}^{*}\right] \text { and } w_{t-1}^{B}=E\left[w \mid w \leq w_{t-1}^{*}\right] .
$$


Conditions (20), (21) and (22) define $w_{0}^{*}$ and $w_{t}^{*}$ as a function of $w_{t-1}^{*}$. A fixed point $w^{*}$ of the sequence gives an equilibrium. This is clear if $w^{*}$ is in ]$w_{\min }, w_{\max }\left[\right.$. Otherwise, say if $w^{*}=w_{\max }$ is a fixed point, then knowing that everybody chooses $B$ at $t-1$, everybody makes the same choice at $t$ : condition (13) is fulfilled and a $B$-equilibrium is obtained. Similarly, $w_{\min }$ is a fixed point iff there is an $A$-equilibrium.

We first prove that $w_{t}^{*} \leq w_{0}^{*}$ for any $t$. Note that $w_{0}^{*}$ is the threshold value associated with expectations $w_{-1}^{A}=w_{-1}^{B}=\bar{w}$. At any subsequent step, the average wage in $A$ can only been larger and that in $B$ be smaller. This implies that the incentives to choose $A$ are larger than at the initial step. Formally note that the inequalities $E[w \mid w \geq x] \geq \bar{w} \geq E[w \mid w \leq x]$ hold whatever $x$. Thus surely $w_{t-1}^{A} \geq \bar{w} \geq w_{t-1}^{B}$. Since $P$ is nondecreasing (20) yields $w_{t}^{*} \leq w_{0}^{*}$.

Assume first $A$ to be more efficient than $B: R^{A}-R^{B} \geq 0$. Since inequalities $E[w \mid w \geq x] \geq x \geq E[w \mid w \leq x]$ always hold, $\left(D^{B} w_{t-1}^{B}-D^{A} w_{t-1}^{A}\right) \leq$ $\left(D^{B}-D^{A}\right) w_{t-1}^{*}$. Thus if $D^{B}-D^{A} \leq 0$, the argument of $P$ in (20) is negative : $w_{t}^{*}=w_{\min }$ whatever $t$, the (unique) $A$-equilibrium is obtained at the opening. If $D^{B}-D^{A}>0$, then $\Delta \geq D^{B}-D^{A} \geq 0$ : the argument of $P$ in (20) is less than $w_{t-1}^{*}$. Thus the sequence $w_{t}^{*}$ decreases as long as it is above $w_{\min }$ : it converges to the $A$-equilibrium.

Assume now that a $B$-equilibrium exists. From Proposition 2, everybody chooses $B$ initially $: w_{0}^{*}=w_{\max }$. Afterwards, $(13)$ gives $w_{1}^{*}=w_{\max }$ : the $B$ equilibrium is reached. Conversely, if a $B$-equilibrium does not exist, surely $w_{1}^{*}<w_{\max }$ : otherwise $w_{1}^{*}=w_{\max } \leq w_{0}^{*}$ implies that $w_{\max }$ is a fixed point of the sequence, namely that there is a $B$-equilibrium.

Finally, assuming $B$ more efficient than $A$, consider the situation without a $B$-equilibrium. Let the function $D^{B} E[w \mid w \leq x]-D^{A} E[w \mid w \geq x]$ be nondecreasing. By induction, using $w_{1}^{*} \leq w_{0}^{*}$, the sequence $w_{t}^{*}$ decreases 
thus converges (since it is bounded). Furthermore, since $w_{1}^{*}<w_{\max }$ (because there is no $B$-equilibrium), the sequence $w_{t}^{*}$ converges to a value strictly less than $w_{\max }$, that is to an equilibrium of type $A B$ or $A$.

\section{References}

Aaron H. [1966] "The Social Security Paradox", Canadian Journal of Economics and Political Sciences, 32, 371-376.

Breyer F. and M. Kolmar [2002] "Are national pension systems efficient if labor is (im)perfectly mobile? Journal of Public Economics, 83, 347-374.

Brown, J.R., O. Mitchell, and J. M. Poterba [2001] "The Role of Real Annuities and Indexed Bonds in an Individual Accounts Retirement Program", in Risk Aspects of Investment-Based Social Security Reform, eds J. Campbell and M. Feldstein, University of Chicago Press for NBER.

Browning E. [1975], 'Why the social insurance budget is too large in a democracy, Economic inquiry 13, 373-388.

Casamatta G., H. Cremer, and P. Pestieau [2000] "The political economy of social security", Scandinavian Journal of Economics 102, 502-522.

Casarico, A. [2000], Pension systems in integrated capital markers, Topics in Economic Analysis and Policy, 1, 1-17.

Cremer H. and P. Pestieau [2003] "Factor mobility and redistribution : a survey", mimeo.

Cremer, H. and P. Pestieau [1998], Social insurance, majority voting and labor mobility, Journal of Public Economics 68; 397-420.

De la Croix D. and M. Doepke [2003] "Inequality and growth : why differential fertility matters", The American Economic Review 93, 1091-1113.

Diamond, P. [1965] "National Debt in a Neoclassical Growth Model" The American Economic Review 55, 1126-1150. 
Epple D., and T. Romer [1991] "Mobility and Redistribution", Journal of Political Economy Vol. 99, No. 4. , pp. 828-858.

Galasso V. and P. Profeta [2002] "The political economy of social security : a survey", European Journal of Political Economy, 18, 1-29.

Gale David [1973] "Pure Exchange Equilibrium of Dynamic Economic Models," Journal of Economic Theory ; 6(1) 12-36.

Homburg S. and W. Richter [1993] "Debt and Public Pension Schemes in the European Community Journal of Economics Suppl. 7, 51-63.

Philipson, T., and G. Becker [1998] "Old-Age Longevity and Mortality Contingent Claims." Journal of Political Economy 106.3 : 550-574.

Samuelson P. [1958], "An exact consumption-loan model of interest with or without the social contrivance of money", Journal of Political economy, 66, 467-482.

Whitehouse E. [2003] "The Value of Pension Entitlements : A Model of Nine OECD Countries, OECD Social, Employment and Migration Working Papers, n 9 . 